3. Carlet C. Vectorial Boolean Functions for Cryptography. Cambridge: Cambridge University Press, 2010. $93 \mathrm{p}$.

4. Панкратова И. А. Свойства компонент некоторых классов векторных булевых функций // Прикладная дискретная математика. 2019. №44. С. 5-11.

\title{
ЛИНЕЙНОЕ РАЗЛОЖЕНИЕ ДИСКРЕТНЫХ ФУНКЦИЙ В ТЕРМИНАХ ОПЕРАЦИИ СДВИГ-КОМПОЗИЦИИ
}

\author{
И. В. Чередник
}

\begin{abstract}
Исследуется операция сдвиг-композиции дискретных функций, возникающая при гомоморфизмах конечных регистров сдвига. Для произвольной функции над конечным полем описаны все возможные представления в виде сдвиг-композиции двух функций, правая из которых линейная. Кроме того, изучена возможность представления произвольной функции над конечным полем сдвиг-композицией трёх функций, в которой обе крайние функции линейные. Доказано, что в случае простого поля для линейных функций, а также для квадратичных функций, линейных по крайней переменной, понятия приводимости и линейной приводимости совпадают.
\end{abstract}

Ключевые слова: дискретные функции, конечные поля, регистр сдвига, сдвигкомпозиция.

\section{Введение}

Пусть $\Omega_{q}$ - конечное множество из $q$ элементов. В данной работе будем использовать множество переменных $\left\{x_{0}, x_{1}, x_{2}, \ldots\right\}$, а множество всех функций $q$-значной логики от переменных $x_{0}, x_{1}, x_{2}, \ldots$ будем обозначать через $F_{q}$. Произвольную функцию $f \in F_{q}$ всегда можно рассматривать как функцию от соответствующего допустимого набора переменных $x_{0}, x_{1}, \ldots, x_{n}$. В работах отечественных криптографов К. Г. Таболова, В. А. Башева, А. Я. Прососова, В. И. Солодовникова и др. была введена и исследована (преимущественно в терминах гомоморфизмов регистров сдвига) операция сдвиг-композиции на множестве всех функций $F_{q}$ :

$$
f\left(x_{0}, \ldots, x_{n}\right) \triangleleft g\left(x_{0}, \ldots, x_{m}\right)=f\left(g\left(x_{0}, \ldots, x_{m}\right), \ldots, g\left(x_{n}, \ldots, x_{n+m}\right)\right) .
$$

В работах перечисленных авторов в разной степени общности и направленности достаточно подробно исследована связь между представлением функции $f$ в виде сдвигкомпозиции $f=g \triangleleft h$ и существованием гомоморфизма регистра сдвига, соответствующего функции $f$, на меньший регистр сдвига, соответствующий функции $g$ (все основные результаты по данной тематике единым образом изложены в [1]). Так, например, в [2] описаны все возможные представления функции $f$ над конечным полем $\mathbb{F}_{q}$ в виде $f=l \triangleleft g$, где $l$ - линейная, что позволило указать все возможные гомоморфизмы регистра сдвига с обратной связью $f$ на линейные регистры сдвига.

В настоящей работе предлагается описание всех возможных представлений произвольной функции $f$ над конечным полем $\mathbb{F}_{q}$ в виде $f=g \triangleleft l$, где $l$-линейная. Кроме того, изучена возможность представления произвольной функции $f$ над конечным полем $\mathbb{F}_{q}$ в виде $f=l_{1} \triangleleft g \triangleleft l_{2}$, где $l_{1}, l_{2}$ - линейные. Доказано, что в случае простого поля для линейных функций, а также для квадратичных функций, линейных по крайней переменной, понятия приводимости и линейной приводимости совпадают. 


\section{1. Основные определения и обозначения}

В данной работе, если не оговорено противное, полагается, что $q=p^{t}$, где $p-$ простое, $t \in \mathbb{N}$, а на множестве $\Omega_{q}$ задана структура поля $\left(\mathbb{F}_{q},+, \cdot\right)$. Известно [3], что каждая функция $f \in F_{q}$ представляется единственным приведённым многочленом из $\mathbb{F}_{q}\left[x_{0}, x_{1}, \ldots\right]$, который для удобства будем отождествлять с функцией $f$.

Для полноты и простоты изложения примеры в данной работе рассматриваются преимущественно в булевом случае, при этом операция сложения в поле $\mathbb{F}_{2}$ выделяется символом «Ф».

Множество всех q-значных функций, которые биективны по первой (последней) переменной, будем обозначать через ${ }^{*} F_{q}\left(F_{q}^{*}\right)$; множество всех $q$-значных функций, которые линейны по первой (последней) переменной, будем обозначать через ${ }^{+} F_{q}\left(F_{q}^{+}\right)$; множество всех функций, сохраняющих константу 0, будем обозначать $\widehat{F}_{q}$. При этом естественны производные обозначения

$$
{ }^{*} F_{q}^{*}=F_{q}^{*} \cap{ }^{*} F_{q}, \quad{ }^{+} F_{q}^{+}={ }^{+} F_{q} \cap F_{q}^{+}, \quad{ }^{*} \widehat{F}_{q}={ }^{*} F_{q} \cap \widehat{F}_{q}, \quad \ldots
$$

Как нетрудно убедиться, каждое из множеств ${ }^{+} F_{q} \subset{ }^{*} F_{q} \subset F_{q}$ образует полугруппу относительно операции $\triangleleft$ с нейтральным элементом $x_{0}$. При этом несложный пример

$$
\left(x_{0} \oplus x_{1}\right) \triangleleft\left(x_{0} \oplus x_{1}\right)=\left(x_{0} \oplus x_{1}\right) \triangleleft\left(x_{0} \oplus x_{1} \oplus 1\right)
$$

показывает, что даже в рамках моноида $\left({ }^{+} F_{q}, \triangleleft\right)$ не всегда возможно производить правое сокращение в равенствах. Однако возможность правого и левого сокращений всё же присутствует в достаточно широких классах практически значимых функций.

Утверждение 1. Множества ${ }^{*} \widehat{F}_{q}, \widehat{F}_{q}^{*},{ }^{*} \widehat{F}_{q}^{*}$ и ${ }^{+} \widehat{F}_{q}, \widehat{F}_{q}^{+},{ }^{+} \widehat{F}_{q}^{+}$образуют моноиды с возможностью левого и правого сокращений.

Будем говорить, что функиия $g$ делит справа функиию $f$, если существует такая функция $h$, для которой выполняется равенство $f=h \triangleleft g$. Для каждой перестановки $\pi$ элементов множества $\Omega_{q}$ произвольная функция $f$ всегда делится справа на $\pi\left(x_{0}\right), \pi(f)$ - такие делители функции $f$ будем называть несобственными. Аналогичным образом определяются соответствующие понятия левой делимости. Если у функции $f$ существует собственный правый, а следовательно, и собственный левый делители, то будем говорить, что функция $f$ приводима.

Замечание 1. Пусть $f \in F_{q}$ и $f(0, \ldots, 0)=c_{f}$. Существует тесная связь между приводимостью $f$ в моноиде $\left(F_{q}, \triangleleft\right)$ и приводимостью $\hat{f}=f-c_{f}$ в подмоноиде $\left(\widehat{F}_{q}, \triangleleft\right)$ : $f=g \triangleleft h \Longleftrightarrow \hat{f}=\left(x_{0}-c_{f}\right) \triangleleft g \triangleleft\left(x_{0}+c_{h}\right) \triangleleft \hat{h}, \quad$ где $\left(x_{0}-c_{f}\right) \triangleleft g \triangleleft\left(x_{0}+c_{h}\right), \hat{h} \in \widehat{F}_{q}$.

Таким образом, исследование приводимости в моноиде $\left(F_{q}, \triangleleft\right)$ во многом сводится к исследованию приводимости в подмоноиде $\left(\widehat{F}_{q}, \triangleleft\right)$.

\section{2. Линейное разложение}

Множество $L_{q}$ всех функций, представимых линейными, но не аффинными многочленами над $\mathbb{F}_{q}$

$$
c_{i_{0}} x_{i_{0}}+c_{i_{1}} x_{i_{1}}+\ldots+c_{i_{k}} x_{i_{k}}: i_{0}<i_{1}<\ldots<i_{k}, k \in \mathbb{N}, c_{i_{0}}, c_{i_{1}}, \ldots, c_{i_{k}} \in \mathbb{F}_{q},
$$

образует коммутативное кольцо $\left(L_{q},+, \triangleleft\right)$, а отображение

$$
c_{i_{0}} x^{i_{0}}+c_{i_{1}} x^{i_{1}}+\ldots+c_{i_{k}} x^{i_{k}} \mapsto c_{i_{0}} x_{i_{0}}+c_{i_{1}} x_{i_{1}}+\ldots+c_{i_{k}} x_{i_{k}}
$$


является изоморфизмом колец $\left(\mathbb{F}_{q}[x],+, \cdot\right)$ и $\left(L_{q},+, \triangleleft\right)[1,2,4]$. Подразумевая этот изоморфизм, далее будем формулировать известные понятия и использовать известные утверждения о делимости многочленов применительно к линейным функциям.

По понятным причинам класс $L_{q}$ является важным с практической точки зрения подмоноидом в $\left(F_{q}, \triangleleft\right)$ и выделение у произвольной функции левых или правых линейных делителей представляется естественной и актуальной задачей. Функцию $f \in F_{q}$ будем называть линейно приводимой справа, если у нее существует собственный правый делитель $l \in L_{q}$. В противном случае функцию $f$ будем называть линейно неприводимой справа. Аналогичным образом определяется левая линейная приводимость функций. Функцию будем называть линейно неприводимой, если она линейно неприводима и справа, и слева.

В. И. Солодовников в [2] описал все возможные левые линейные разложения для произвольной функции из $\widehat{F}_{q}$.

Теорема 1 [2]. Произвольная функция $f \in \widehat{F}_{q}$ однозначно представляется в виде

$$
f=\sum_{\substack{1 \leqslant i_{1}<\ldots<i_{k}, 1 \leqslant a_{0}, a_{1}, \ldots, a_{k}<q}} l_{i_{1}, \ldots, i_{k} ; a_{0}, a_{1}, \ldots, a_{k}} \triangleleft x_{0}^{a_{0}} x_{i_{1}}^{a_{1}} \ldots x_{i_{k}}^{a_{k}},
$$

где $l_{i_{1}, \ldots, i_{k} ; a_{0}, a_{1}, \ldots, a_{k}} \in L_{q}$ для всех $1 \leqslant i_{1}<\ldots<i_{k}, 0<a_{0}, a_{1}, \ldots, a_{k}<q$.

При этом все левые линейные делители функции $f$ исчерпываются делителями $l=$ НОД $\left(l_{i_{1}, \ldots, i_{k} ; a_{0}, a_{1}, \ldots, a_{k}}: 1 \leqslant i_{1}<\ldots<i_{k}, 0<a_{0}, a_{1}, \ldots, a_{k}<q\right)$. В частности, функция $f$ линейно неприводима слева в том и только в том случае, когда $l=x_{0}$.

Линейную функцию вида $x_{0}+a_{1} x_{i_{1}}+\ldots+a_{k} x_{i_{k}}$ будем называть унитарной no переменной $x_{0}$.

Следствие 1 [2]. Произвольная функция $f \in \widehat{F}_{q}$ однозначно представляется в виде $f=x_{s} \triangleleft l \triangleleft g$, где $x_{s}$ - крайняя левая переменная, от которой $f$ зависит существенным образом; $l \in L_{q}-$ унитарная по переменной $x_{0} ; g$-линейно неприводимая слева.

Для описания правых делителей потребуются дополнительные обозначения. Известно $[5,6]$, что в случае $q=p^{t}$, где $p$-простое, $t>1$, степень нелинейности приведённого одночлена $x^{a} \in \mathbb{F}_{q}[x], a<q$, лучше оценивать не самим числом $a$, а его p-ичным весом

$$
\|a\|_{p}=a_{0}+\ldots+a_{t-1}
$$

определяемым из р-ичного представления

$$
a=a_{0}+\ldots+a_{t-1} p^{t-1}, 0 \leqslant a_{0}, \ldots, a_{t-1}<p .
$$

В связи с этим одночлен $x^{a}$ часто расписывают в виде $x^{a_{0}} \ldots x^{p^{t-1}} a_{t-1}$, а произвольный приведённый моном $\mathbf{x}^{\mathbf{a}}=x_{0}^{a_{0}} \ldots x_{n}^{a_{n}}$, где $0 \leqslant a_{0}, \ldots, a_{n}<q$ и $a_{i}=a_{i 0}+\ldots+a_{i t-1} p^{t-1}$, $i \in\{0, \ldots, n\},-$ в виде

$$
\mathbf{x}^{\mathbf{a}^{\mathbf{p}}}=x_{0}^{a_{00}} \ldots x_{0}^{p^{t-1} a_{0 t-1}} \ldots x_{n}^{a_{n 0}} \ldots x_{n}^{p^{t-1} a_{n t-1}}
$$

подразумевая при этом $\mathbf{a}^{\mathbf{p}}=\left(a_{00}, \ldots, a_{0 t-1}, \ldots, a_{n 0}, \ldots, a_{n t-1}\right)$.

Отношение градуированного лексикографического порядка на $\mathbb{N}_{0}^{(n+1) t}$ индуцирует отношение порядка на множестве приведенных мономов из $\mathbb{F}_{q}\left[x_{0}, \ldots, x_{n}\right]$

$$
\mathbf{x}^{\mathbf{a}^{\mathbf{p}}} \geqslant \mathbf{x}^{\mathrm{b}^{\mathbf{p}}} \Leftrightarrow \mathbf{a}^{\mathbf{p}} \geqslant \text { grlex } \mathbf{b}^{\mathbf{p}},
$$


при котором мономы сначала упорядочиваются по степени нелинейности, а мономы одной степени нелинейности упорядочиваются «лексикографически» при условии

$$
x_{0}>\ldots>x_{0}^{p^{t-1}}>\ldots>x_{n}>\ldots>x_{n}^{p^{t-1}} .
$$

При простом $q$ введённое отношение порядка совпадает со стандартным градуированным лексикографическим порядком на множестве всех приведённых мономов из $\mathbb{F}_{q}\left[x_{0}, \ldots, x_{n}\right]$.

Пусть

$$
\mathbf{x}^{\mathbf{a}^{\mathbf{p}}}=x_{i_{0}}^{a_{00}} \ldots x_{i_{0}}^{p^{t-1} a_{0 t-1}} \ldots x_{i_{k}}^{a_{k 0}} \ldots x_{i_{k}}^{p^{r-1} a_{k r-1}} x_{i_{k}}^{p^{r} a_{k r}}, \quad 0<a_{k r}<p .
$$

Тогда мономы

$$
x_{i_{0}}^{a_{00}} \ldots x_{i_{0}}^{p^{t-1} a_{0 t-1}} \ldots x_{i_{k}}^{a_{k 0}} \ldots x_{i_{k}}^{p^{r-1} a_{k r-1}} x_{i_{k}}^{p^{r}\left(a_{k r}-1\right)} x_{i_{k}+j}^{p^{r}}, \quad j \geqslant 1,
$$

и только их будем называть линейно связанными с мономом $\mathrm{x}^{\mathbf{a}^{\mathbf{p}}}$.

Теорема 2. Произвольная функция $f \in F_{q}$ однозначно представляется в виде

$$
f=c+\sum_{i=0}^{m} c_{i} \mathbf{x}^{\mathbf{a}_{i}^{\mathbf{p}}} \triangleleft l_{i}\left(x_{0}, \ldots\right)
$$

где $c \in \Omega_{q} ; \mathbf{x}^{\mathbf{a}_{0}^{\mathrm{p}}}>\ldots>\mathbf{x}^{\mathbf{a}_{m}^{\mathrm{p}}}$ - убывающая последовательность линейно несвязанных мономов, и для каждого $i \in\{0, \ldots, m\}$ коэффициент $c_{i} \in \Omega_{q}$ отличен от 0 , а $l_{i}\left(x_{0}, \ldots\right)-$ линейная функция, унитарная по переменной $x_{0}$.

При этом если $f$ существенно зависит от переменной $x_{0}$, то все правые линейные делители функции $f$ исчерпываются делителями НОД $\left(l_{0}, \ldots, l_{m}\right)$. В частности, функция $f$ линейно неприводима справа в том и только в том случае, когда НОД $\left(l_{0}, \ldots, l_{m}\right)=x_{0}$.

Следствие 2. Произвольная функция $f \in F_{q}$ однозначно представляется в виде $f=x_{s} \triangleleft g \triangleleft l$, где $x_{s}$ - крайняя левая переменная, от которой $f$ зависит существенным образом; $g$ - линейно неприводимая справа; $l \in L_{q}-$ унитарная по переменной $x_{0}$.

Замечание 2. Представление, доказанное в теореме 2, существенным образом зависит от условий $x_{0}>\ldots>x_{0}^{p^{t-1}}>\ldots \ldots>x_{n}>\ldots>x_{n}^{p^{t-1}}$. Так, для функции

$$
f=x_{0}^{3}+x_{0}^{2} x_{2}+x_{0} x_{1}^{2}+x_{0} x_{2}^{2}
$$

над полем $\mathbb{F}_{4}$ при условии $x_{0}>x_{0}^{2}>x_{1}>x_{1}^{2}>x_{2}>x_{2}^{2}$ справедливо разложение

$$
f=x_{0} x_{0}^{2} \triangleleft\left(x_{0}+x_{1}+x_{2}\right)+x_{1} x_{1}^{2} \triangleleft\left(x_{0}+x_{1}\right)+x_{0}^{2} x_{1} \triangleleft x_{0},
$$

а при условии $x_{0}^{2}>x_{0}>x_{1}^{2}>x_{1}>x_{2}^{2}>x_{2}$ - разложение

$$
f=x_{0}^{2} x_{0} \triangleleft\left(x_{0}+x_{2}\right)+x_{0} x_{1}^{2} \triangleleft x_{0}+x_{2}^{2} x_{2} \triangleleft x_{0} .
$$




\section{3. Особенности двустороннего линейного разложения}

Теорема 3. Пусть $q$ простое и функция $f \in F_{q}$ делится слева на $l_{1} \in L_{q}$, а справа на $l_{2} \in L_{q}$. Тогда если $l_{1}$ и $l_{2}$ взаимно просты, то справедливо разложение

$$
f=l_{1} \triangleleft g \triangleleft l_{2} .
$$

Если, дополнительно, $l_{1}$ и $l_{2}$ - максимальные левый и правый делители функции $f$, то $g$ - линейно неприводимая.

Замечание 3. Условие простоты $q$ является существенным в теореме 3. Так, например, если $q=p^{t}, t \geqslant 2$ и $\alpha \in \mathbb{F}_{q} \backslash \mathbb{F}_{p}$, то $\alpha^{p} \neq \alpha$ и, очевидно, линейные функции $x_{0}+\alpha x_{1}, x_{0}+\alpha^{p} x_{1}$ взаимно просты. Однако при этом справедливы разложения

$$
\left(x_{0}+\alpha^{p} x_{1}^{p}\right) \triangleleft x_{0}^{p}=x_{0}^{p}+\alpha^{p} x_{1}^{p}=x_{0}^{p} \triangleleft\left(x_{0}+\alpha x_{1}\right)
$$

и легко убедиться в невозможности представления

$$
x_{0}^{p}+\alpha^{p} x_{1}^{p}=\left(x_{0}+\alpha^{p} x_{1}^{p}\right) \triangleleft g \triangleleft\left(x_{0}+\alpha x_{1}\right) .
$$

Замечание 4. Условие взаимной простоты левого и правого линейных делителей является существенным в теореме 3. Так, например, для булевой функции

$$
f=\left(x_{0} \oplus x_{1}\right) \triangleleft x_{0} x_{1} \triangleleft\left(x_{0} \oplus x_{1}\right) \oplus\left(x_{0} \oplus x_{1}\right)
$$

справедливы разложения

$$
\begin{aligned}
& f=\left(x_{0} \oplus x_{1}\right) \triangleleft\left(x_{0} x_{1} \oplus x_{0} x_{2} \oplus x_{1} x_{2} \oplus x_{0} \oplus x_{1}\right)=\left(x_{0} \oplus x_{1}\right) \triangleleft g_{1}, \\
& f=\left(x_{0} x_{1} \oplus x_{1} x_{2} \oplus x_{0}\right) \triangleleft\left(x_{0} \oplus x_{1}\right)=g_{2} \triangleleft\left(x_{0} \oplus x_{1}\right),
\end{aligned}
$$

но при этом $g_{1}$-линейно неприводимая справа, $g_{2}$ - линейно неприводимая слева, а потому невозможно представление

$$
f=\left(x_{0} \oplus x_{1}\right) \triangleleft g \triangleleft\left(x_{0} \oplus x_{1}\right) .
$$

\section{4. Квадратичные функции над простым полем}

Ввиду изоморфизма колец $\left(L_{q},+, \triangleleft\right)$ и $\left(\mathbb{F}_{q},+, \cdot\right)$ описание всех линейных делителей произвольной функции $f \in L_{q}$ равносильно определению канонического разложения соответствующего многочлена.

Для описания линейных делителей произвольной квадратичной функции можно использовать результаты теорем 2 и 3 и, как показывает следующий результат, в случае линейных по крайней переменной квадратичных функций над простым полем это позволяет описать вообще все возможные делители.

Теорема 4. Если $q-$ простое число, то для композиции $f \triangleleft g$ произвольных функций $f \in F_{q}$ и $g \in{ }^{+} F_{q} \cup F_{q}^{+}$справедливы следующие утверждения:

1) $\operatorname{deg}(f \triangleleft g)=0$ тогда и только тогда, когда $\operatorname{deg} f=0$;

2) $\operatorname{deg}(f \triangleleft g)=1$ тогда и только тогда, когда $\operatorname{deg} f=\operatorname{deg} g=1$;

3) $\operatorname{deg}(f \triangleleft g)=2$ тогда и только тогда, когда либо $\operatorname{deg} f=1$ и $\operatorname{deg} g=2$, либо $\operatorname{deg} f=2$ и $\operatorname{deg} g=1$.

Замечание 5. Пункт 2 теоремы 4 в частном случае $q=2$ был доказан В. И. Солодовниковым в 1978 г. [1].

Замечание 6. Простой пример

$$
\left(x_{1} x_{4} \oplus x_{2} x_{3} x_{4}\right) \triangleleft\left(x_{0} \oplus x_{1} x_{2} \oplus x_{2}\right)=x_{1} x_{4} \oplus x_{1} x_{5} x_{6} \oplus x_{1} x_{6} \oplus x_{3} x_{4} \oplus x_{3} x_{5} x_{6} \oplus x_{3} x_{6}
$$

показывает, что уже в случае кубических функций понятие приводимости становится шире понятия линейной приводимости. 


\title{
ЛИТЕРАТУРА
}

1. Солодовников В.И. Регистры сдвига и криптоалгоритмы на их основе. LAP LAMBERT Academic Publishing, 2017.

2. Солодовников В. И. Гомоморфизмы регистров сдвига в линейные автоматы // Дискретная математика. 2008. № 4. С. 87-101.

3. Лидл Р., Нидеррайтер Г. Конечные поля. М.: Мир, 1988.

4. Солодовников В. И. Гомоморфизмы двоичных регистров сдвига // Дискретная математика. 2005. № 1. С. 73-88.

5. Кузвмин А.С., Нечаев А.А., Шишкин В.А. Бент- и гипербент-функции над конечным полем // Труды по дискретной математике. 2007. № 10. С. 97-122.

6. Черемушкин A. В. Аддитивный подход к определению степени нелинейности дискретной функции // Прикладная дискретная математика. 2010. № 2. С. 22-33.

\section{О ВЗАИМОСВЯЗИ МЕЖДУ КВАТЕРНАРНЫМИ И БУЛЕВЫМИ БЕНТ-ФУНКЦИЯМИ ${ }^{1}$}

\author{
А. С. Шапоренко
}

Исследуются кватернарные бент-функции вида $f: \mathbb{Z}_{4}^{n} \rightarrow \mathbb{Z}_{4}$. Показано представление коэффициентов Уолша - Адамара кватернарной функции через коэффициенты двух булевых функций. Получено, что любая кватернарная бент-функция является регулярной. Изучается связь кватернарных бент-функций от одной и двух переменных с булевыми бент-функциями от двух и четырёх переменных соответственно.

Ключевые слова: кватернарные функции, булевы функиии, регулярные бентфункиии.

Пусть $\langle x, y\rangle$-скалярное произведение векторов, где суммирование производится по модулю 2, а $x . y$ - скалярное произведение векторов с суммированием по модулю 4.

Преобразованием Уолша-Адамара булевой функиии $f$ от $n$ переменных называется целочисленная функция $W_{f}(x)$, заданная на множестве $\mathbb{Z}_{2}^{n}$ равенством

$$
W_{f}(x)=\sum_{y \in \mathbb{Z}_{2}^{n}}(-1)^{\langle x, y\rangle \oplus f(y)} .
$$

Булева функция $f$ от $n$ ( $n$ - чётное) переменных называется бент-функиией, если $\left|W_{f}(x)\right|=2^{n / 2}$ для любого $x \in \mathbb{Z}_{2}^{n}$.

Функция $g: \mathbb{Z}_{4}^{n} \rightarrow \mathbb{Z}_{4}$ называется кватернарной функиией от $n$ переменных [1]. Преобразование Уолша - Адамара кватернарной функиии g определяется следующим образом:

$$
W_{g}(x)=\sum_{y \in \mathbb{Z}_{4}^{n}} i^{x \cdot y+g(y)}
$$

Здесь «+» означает сложение по модулю 4.

Кватернарная функция $g$ от $n$ переменных называется бент-функиией, если $\left|W_{g}(x)\right|=4^{n / 2}$ для любого $x \in \mathbb{Z}_{4}^{n}$.

\footnotetext{
${ }^{1}$ Работа выполнена при финансовой поддержке РФФИ (проект № 18-07-01394), Министерства образования и науки (Задание № 1.13559.2019/13.1 и Программа 5-100).
} 